\title{
Evaluation of the mechanical behaviour of different devices for canine retraction
}

Antônio Carlos de Oliveira Ruellas' ${ }^{1}$, Matheus Melo Pithon², Rogério Lacerda dos Santos ${ }^{3}$

Objective: To mechanically evaluate different systems used for canine retraction.

Methods: Three different methods for partial canine retraction were evaluated: retraction with elastic chain directly attached to bracket; elastic chain connected to bracket hook and with sliding jig activated with the aid of an elastic chain attached to a mini-implant. For this evaluation, a Typodont was adapted to simulate the desired movements when exposed to a heat source. After obtaining the measurements of the movements, statistical analysis was performed.

Results: The mini-implant/sliding jig system (Groups M 0.018-in and M 0.019 x 0.026-in) favored less extrusion and distal inclination of the canines in the retraction stage $(\mathrm{p}<0.005)$. Meanwhile, the retraction system with elastic chain directly attached to the orthodontic brackets (Groups C 0.018 -in and $0.019 \times 0.026$-in) favored greater inclination and extrusion than the others, followed by the system of elastic chain attached to the hook (Groups G 0.018-in and $0.019 \times 0.026$-in).

Conclusions: Canine retraction with the mini-implant/sliding jig system showed the best mechanical control. The worst results were observed with a 0.018 archwire when the elastic chain was attached to the bracket.

Keywords: Corrective Orthodontics. Canine tooth. Malocclusion.

${ }^{1}$ Associate Professor, Department of Orthodontics, Federal University of Rio de Janeiro.

${ }^{2}$ Associate Professor, Southwest Bahia University.

${ }^{3}$ Professor of Orthodontics, Federal University of Campina Grande.
How to cite this article: Ruellas ACO, Pithon MM, Santos RL. Evaluation of the mechanical behaviour of different devices for canine retraction. Dental Press J Orthod. 2012 May-June;17(3):83-7.

Submitted: March 05, 2009 - Revised and accepted: August 16, 2009

" The authors report no commercial, proprietary or financial interest in the products or companies described in this article.

Contact address: Antônio Carlos de Oliveira Ruellas

Av. Professor Rodolpho Paulo Rocco, 325 - Ilha do Fundão - Zip code: 21941-617

Rio de Janeiro/RJ - Brazil - E-mail: antonioruellas@yahoo.com.br 


\section{INTRODUCTION}

During orthodontic treatment, precise diagnosis and consequent correct treatment plan presents a high degree of difficulty and complexity.

When defining the treatment plan, a significant percentage of malocclusions, such as discrepancies between tooth and maxillary sizes, and discrepancies between the bone bases normally results in extraction therapies. ${ }^{6,5}$

Space closure must be performed in a planned and adequate manner. ${ }^{7}$ For this purpose, according to orthodontic planning, the canine teeth will be partially or completely retracted, and afterwards, the remaining spaces will be closed by means of a specific system of force. ${ }^{1}$

The choice of the mechanism for canine retraction requires profound knowledge of the characteristics presented by these devices, such as: maximum tooth movement, control of vertical, horizontal and rotational forces, conserving the integrity of the root and circumjacent tissues. ${ }^{2,3,4,7,8}$

Based on this premise, the aim of this study was to perform a mechanical evaluation of the different systems used for canine retraction, thus making it possible to explain to the orthodontist which would be the best system to develop this function.

\section{MATERIAL AND METHODS}

To conduct the experiment, a wax Typodont was mounted in normal occlusion to allow tooth movement when exposed to a heat source.

Once the Typodont was adapted, the teeth were mounted in a Class I malocclusion with bimaxillary protrusion. This malocclusion was selected because extraction of the first premolars is the therapy routinely used in these cases, followed by retraction of the canines and incisors.

After the Typodont was mounted, orthodontic brackets were bonded according to the edgewise slot $0.022 \times 0.030$-in technique, which would serve as support for the application of orthodontic mechanics.

After the orthodontic appliance was mounted, the Typodont was fixed on a rigid rod, which enabled the occlusal plane to remain parallel to the ground and perpendicular to a $30 \mathrm{~cm}$ long ruler, the purpose of which was to measure the extrusion of the incisors that would occur during retraction (Fig 1).
Three different methods of partial canine retraction were evaluated in two different types of orthodontic arches, therefore the groups were divided as follows:

Group C 0.018-in: Retraction performed with elastic chain directly connected to the bracket in a 0.018-in stainless steel archwire (Fig 2).

Group G 0.018-in: Retraction performed with elastic chain connected to the bracket hook in a 0.018-in stainless steel archwire (Fig 3).

Group M 0.018-in: Retraction performed with a sliding jig activated with elastic chain attached to a mini-implant in a 0.018-in stainless steel archwire (Fig 4).

Group C $0.019 \times$ 0.026-in: Retraction performed with elastic chain directly connected to the bracket in a $0.019 \times 0.026$-in stainless steel archwire.

Group G 0.019 x 0.026-in: Retraction performed with elastic chain connected to the bracket hook in a $0.019 \times 0.026$-in stainless steel archwire.

Group M 0.019 x 0.026-in: Retraction performed with a sliding jig activated with elastic chain attached to a mini-implant in a $0.019 \times 0.026-$ in stainless steel archwire.

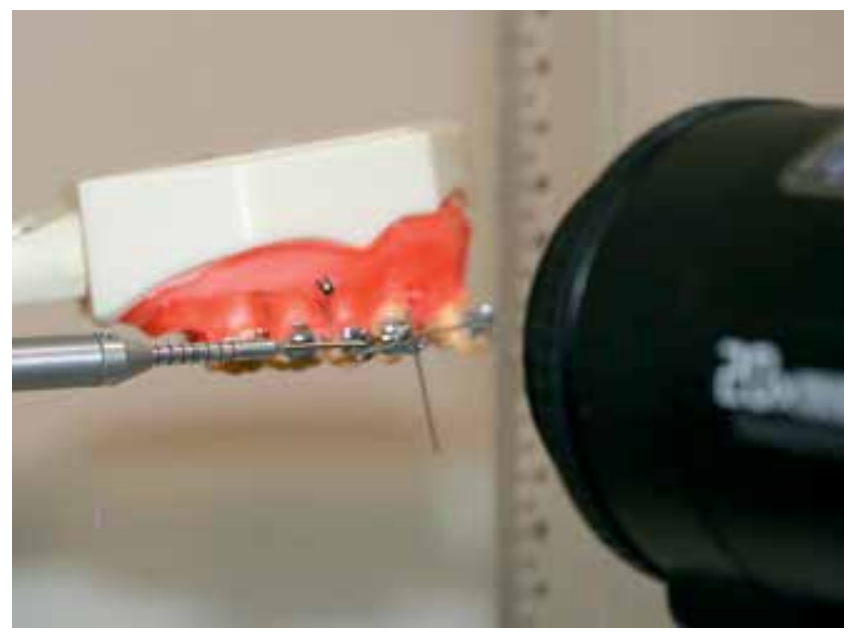

Figure 1 - Typodont in position during the canine retraction assay. 

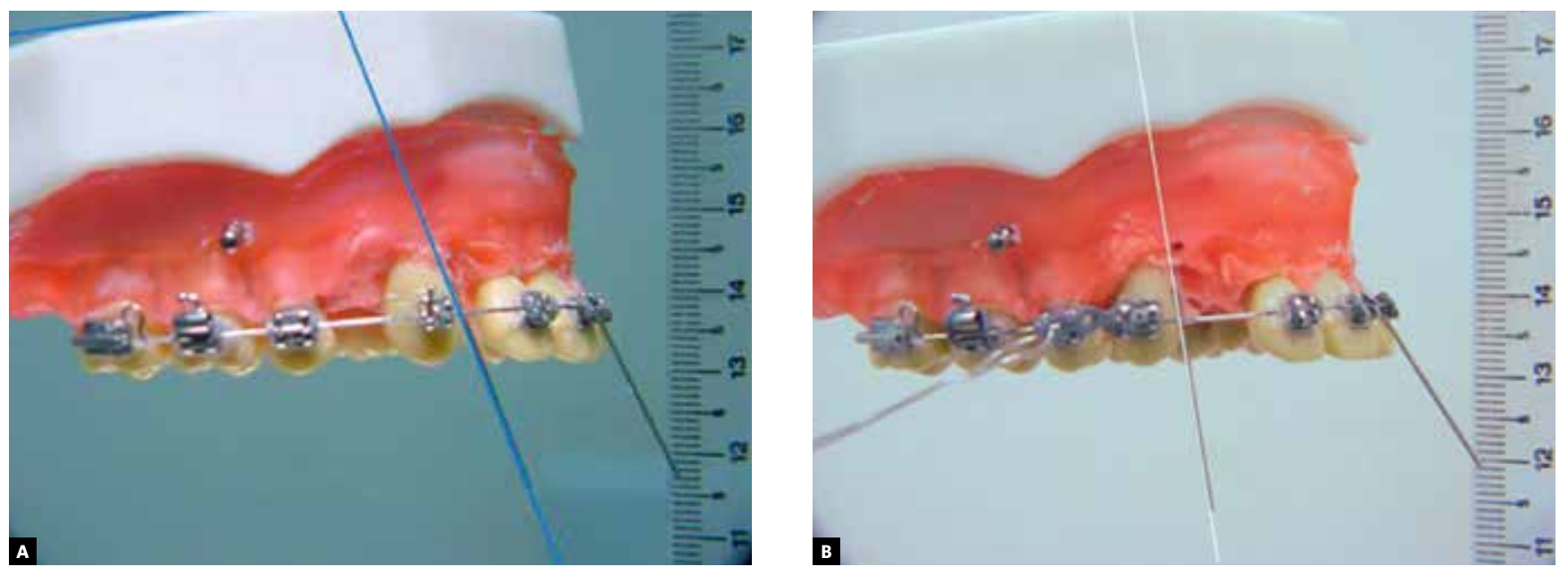

Figure 2 - A) Canine position before retraction with elastic placed on bracket wing; B) retracted canine.
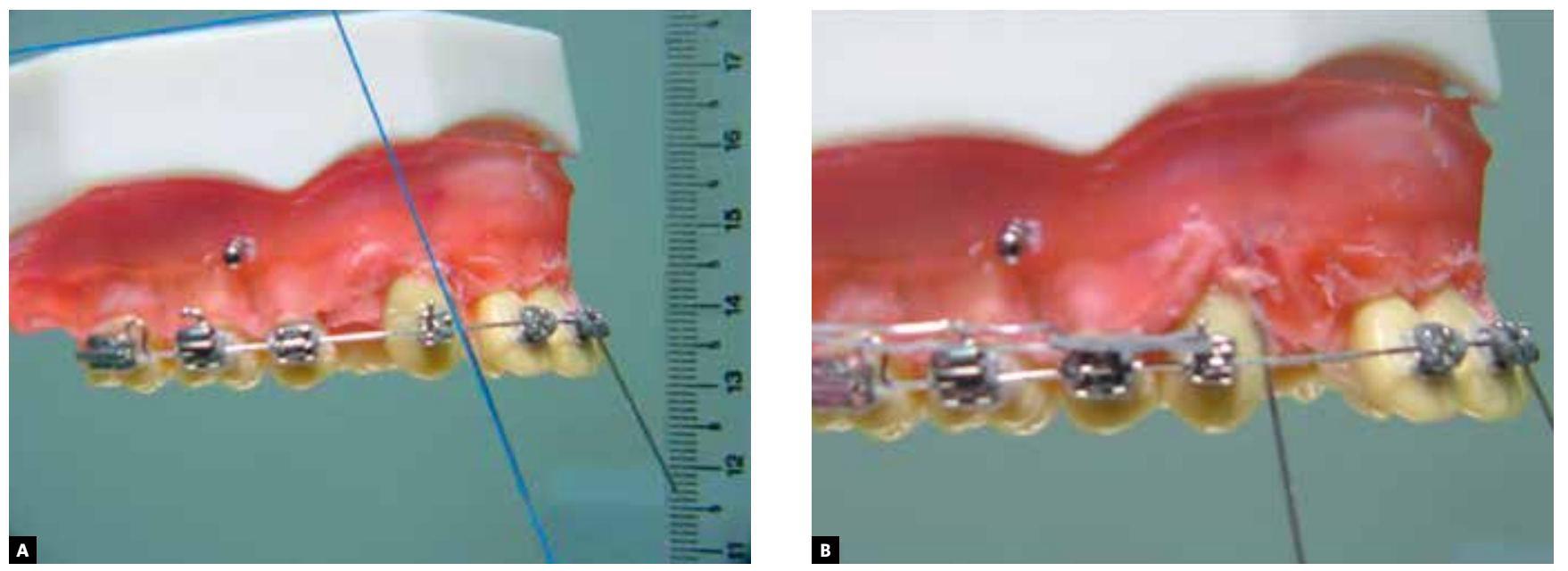

Figure 3 - A) Canine position before retraction with elastic placed on welded hook; B) retracted canine.
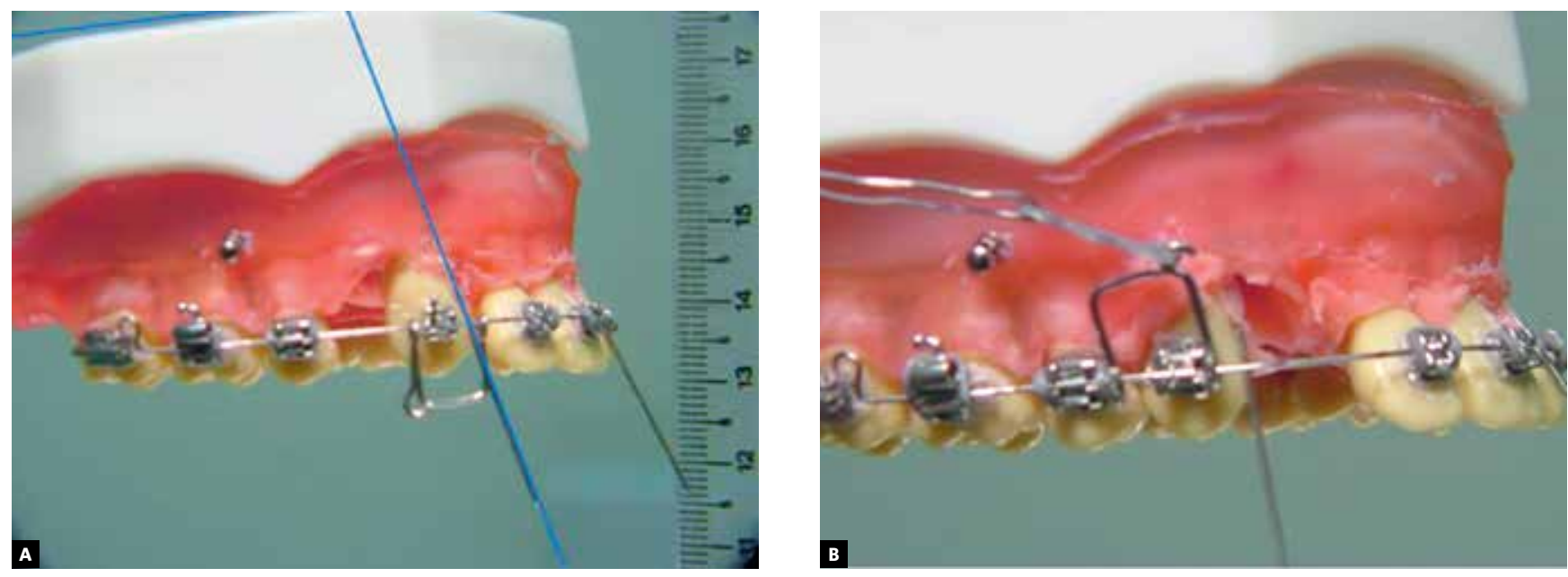

Figure 4 - A) Canine position before retraction with sliding jig; B) retracted canine. 
Activation of the elastic chain was performed with the aid of a dynamometer, whose purpose was to activate and measure the force necessary for retraction.

The canines were retracted to an extension of 8 $\mathrm{mm}$, and in each set, 15 repetitions were performed, thus enabling the groups to be statistically evaluated.

After data collection, statistical analysis was performed using the program SPSS 13.0 (SPSS Inc., Chicago, Illinois, USA). The amount of incisor extrusion, post-retraction canine tipping and force for retraction obtained in millimeters, angle and gram-force were submitted to the analysis of variance (ANOVA) to determine whether there were any statistical differences among the groups, and after this Tukey's test was performed.

\section{RESULTS}

The results demonstrated that the mini-implant/sliding jig system (Groups M 0.018-in and M $0.019 \times 0.026$-in) favored less extrusion of the incisors (Table 1) and greater distal tipping (Table 2) of the canines in the retraction stage $(\mathrm{p}<0.005)$. The retraction system with elastic chain directly attached to orthodontic brackets (Groups C 0.018-in and $0.019 \times 0.026$-in) favored greater canine tipping

Table 1 - Amount of extrusion among groups.

\begin{tabular}{|c|c|c|c|}
\hline Groups & Mean (mm) & s.d. & Statistical analysis ${ }^{\star}$ \\
\hline C 0.018-in & 2.6 & 0.4 & A \\
\hline G 0.018-in & 0.6 & 0.1 & B \\
\hline M 0.018-in & 0.1 & 0.2 & C \\
\hline C $0.019 \times 0.026$-in & 1.9 & 0.3 & D \\
\hline G $0.019 \times 0.026$-in & 0.5 & 0.1 & B \\
\hline M 0.019 x 0.026-in & 0.1 & 0.2 & C \\
\hline
\end{tabular}

^ Equal letters mean absence of statistical differences.

Table 2 - Values of angulation acquired by canines post retraction.

\begin{tabular}{lccc} 
Groups & Mean (mm) & s.d. & Statistical analysis \\
\hline C 0.018-in & -12 & -3 & $A$ \\
G 0.018-in & -10 & -3 & $A$ \\
M 0.018-in & -2 & -2 & $B$ \\
C 0.019 x 0.026-in & -6 & -2 & $C$ \\
G 0.019 x 0.026-in & -5 & -3 & $C$ \\
M 0.019 x 0.026-in & -1 & -2 & $B$ \\
\hline
\end{tabular}

* Equal letters mean absence of statistical differences. and incisor extrusion than the other groups, followed by the system of elastic chain attached to the hook (Groups G 0.018-in and 0.019 x 0.026-in).

However, regarding inclination, there were no statistical differences among the systems in which the elastic chain was placed directly onto the bracket and in which it was placed on the hook welded to the bracket ( $p>0.005$ ) (Table 2).

When the values of force required for canine retraction was evaluated, the Groups $\mathrm{C}$ 0.018-in and $\mathrm{G}$ 0.018-in required lower forces. Higher forces were required in Group M 0.019 x 0.026-in.

\section{DISCUSSION}

Precise knowledge of the mechanical implications of orthodontic appliances is a decisive factor for success or failure of the treated cases. The stage of retraction of the teeth is characterized as one of the most critical stages, requiring precise mechanical knowledge, thereby avoiding undesirable movements and loss of control during treatment.

Based on this premise, the aim of the present study was to evaluate the mechanical behavior of different methods of canine retraction, thus making it possible to provide the orthodontist with information which can be applied in daily clinical practice.

For this purpose a new methodology was developed, in which a dental Typodont was used, with teeth mounted on a heat sensitive wax base. This method was based on a Typodont method, which enabled evaluation of the extrusion and angulation movements.

When the incisor extrusion occurred during retraction was compared, Group C 0.018-in presented greater extrusion than the others. This could be justified by the more occlusal position of the force vector, so that it remained more distant from the

Table 3 - Force required for canine retraction in the different systems.

\begin{tabular}{lc}
\multicolumn{1}{c}{ Groups } & Force variation (N) \\
\hline C 0.018-in & $150-320$ \\
G 0.018-in & $160-310$ \\
M 0.018-in & $160-370$ \\
C $0.019 \times 0.026$-in & $160-390$ \\
G $0.019 \times 0.026$-in & $155-380$ \\
M 0.019 x 0.026-in & $165-430$ \\
\hline
\end{tabular}


center of resistance; and the greater flexibility presented by the 0.018 -in steel wire in comparison with $0.019 \times 0.026$-in wire.

This provided greater distal tipping of the canine, and consequently, greater extrusion of the incisors. This fact may compromise esthetics with greater exposure of the incisors and gummy smile.

Group C $0.019 \times 0.026$-in was ranked second as the system in which most extrusion occurred. The discrete reduction in extrusion in comparison with Group C 0.018-in was due to the greater stiffness of the $0.019 \times 0.026$-in arch, favoring the fact that the results of the two groups differed statistically $(\mathrm{p}<0.05)$.

Groups that were retracted with the sliding jig attached to mini-implants (M 0.018-in and 0.019 x 0.026in) had the lowest values of incisor extrusion.

This fact is related to the proximity of the force vector to the center of resistance of the tooth, which allows better control of the distal tipping of the canine. The caliper of the arch was not shown to be important, since no statistical differences occurred between these two groups ( $\mathrm{p}>0.05)$.

Intermediate extrusion values were obtained with regard to retraction with elastic chain attached to a hook welded to the bracket (Groups G 0.018-in and $0.019 \times 0.026$-in). These values were due to the greater approximation of the force vector to the center of resistance, not as close as occurred in Groups $\mathrm{M}$ (0.018-in and $0.019 \times 0.026$-in) and not as distant as in Groups C (0.018-in and $0.019 \times 0.026$-in).

With regard to the angulation that the canines underwent during retraction, Groups M 0.018-in and $0.019 \times 0.026$-in, were shown to have less inclination than the other groups without statistical difference among them. Groups C 0.018-in and G 0.018-in were inclined with greater amplitude than the others, however, without statistical differences among them ( $p>0.05)$. The conjunction of smaller arches (0.018-in) with a greater distance from the center of resistance was responsible for these results.

It is clinically important, whenever possible, to approximate the force vector to the center of resistance of the tooth to the maximum extent. Among the resources for this purpose, the accessory could be bonded in a more cervical direction, using longer hooks welded to the distal winglet of the bracket and sliding jig.
Another evaluated factor was the required force for retraction. Groups C 0.018-in and G 0.018-in, required lower forces than the other groups. Greater forces were required for Group M 0.019 x 0.026-in, as a result of the friction generated with the use of this arch. The groups in which retraction was performed with arch 0.018-in required lower forces than those performed in a rectangular arch. The group with the sliding jig probably required greater force due to the fact that this system produces more bodily movement (translation) than distal tipping. The greatest difficulty for bodily movement is the great amount of force necessary to do that.

\section{CONCLUSIONS}

By conducting this study, it could be concluded that:

" Thicker arches presented greater vertical control and less distal tipping of the canines during retraction.

» The use of the sliding jig attached to a miniimplant approximated the force vector to the center of resistance of the tooth, providing better mechanical control.

\section{REFERENCES}

1. Burstone CJ. The segmented arch approach to space closure. Am J Orthod. 1982 Nov;82(5):361-78

2. Deguchi T, Imai M, Sugawara Y, Ando R, Kushima K, Takano-Yamamoto T. Clinical evaluation of a low-friction attachment device during canine retraction. Angle Orthod. 2007 Nov;77(6):968-72.

3. Farrant SD. An evaluation of different methods of canine retraction. $\mathrm{Br} J$ Orthod. 1977 Jan;4(1):5-15.

4. Giancotti A, Greco M. Sliding mechanics in extraction cases with a bidimensional approach. Prog Orthod. 2010;11(2):157-65.

5. Noroozi H. A formula to determine the amount of retraction of mandibular canines. Angle Orthod. 2000 Apr;70(2):154-6.

6. Ricketts RM. Bioprogressive therapy as an answer to orthodontic needs. Part II. Am J Orthod. 1976 Oct;70(4):359-97.

7. Shpack N, Davidovitch M, Sarne O, Panayi N, Vardimon AD. Duration and anchorage management of canine retraction with bodily versus tipping mechanics. Angle Orthod. 2008 Jan;78(1):95-100.

8. Skoularikis P, Wichelhaus A, Sander FG. Clinical experience with a new superelastic Ni-Ti-stainless steel retraction spring. World J Orthod. 2008 Spring;9(1):48-51. 